\title{
Photodiode based on epitaxial silicon with high sensitivity at the wavelength $254 \mathrm{~nm}$
}

\author{
Yu. Dobrovolskyi ${ }^{1, *}$, L. Pidkamin ${ }^{1}$, V. Brus ${ }^{2}$, V. Kuzenko ${ }^{2}$ \\ ${ }^{1}$ Scientific and developed firm "Tensor" Ltd, 58013 Chernivtsi, Ukraine \\ ${ }^{2}$ Bukovinian State Financial and Economics University, 58000 Chernivtsi, Ukraine \\ ${ }^{*}$ Correspondence author phone: +38 (03722) 7-87-21; e-mail: yuriydrg@ukr.net
}

\begin{abstract}
A mathematical model of the construction of silicon photodiode based on epitaxial structure enabling to regulate the absorption edge of silicon in the long-wave spectral range is presented. The suggested model allows calculating the construction that possesses low sensitivity for the wavelengths larger than $600 \mathrm{~nm}$ and maximal values near the wavelength $254 \mathrm{~nm}$.
\end{abstract}

Keywords: photodiode, silicon, sensitivity, ultraviolet radiation.

Manuscript received 12.01.14; revised version received 26.07.14; accepted for publication 16.09.14; published online 30.09.14.

\section{Introduction}

Silicon is hi-tech material for obtaining semiconductor devices including photodiodes. In this research, the possibility of using silicon photodiodes in ultraviolet (UV) radiometers is considered.

In terrestrial UV radiometers, the photodiodes with sensitivity within the range 200 to $380 \mathrm{~nm}$ are used. Such photodiodes have to be characterized by high sensitivity and a wide dynamic range. The photodiodes based on gallium phosphide, gallium nitride and zinc selenide meet these requirements. Technology of producing these photodiodes is more sophisticated than, e.g., technology of silicon photodiodes.

Within the spectral range 200 to $380 \mathrm{~nm}$, the sensitivity of silicon photodiode reaches high values. It can exceed the sensitivity of photodiodes based on gallium phosphide or zinc selenide [1]. On the other hand, it is known that photodiodes based on silicon are sensitive in the near infrared spectral range. However, this sensitivity cannot be confined to zero by using light filters.

We have already considered and suggested constructions of photodiodes based on silicon epitaxial structures $[2,3]$. The sensitivity of these structures was determined in the visible spectral range. That is why it was suggested to choose the thickness of the epitaxial layer equal to the absorption depth of visible light. The research work of A. Malik [4,5] was used as the foundation for the development of photodiodes based on gallium phosphide for ultraviolet spectrum. Later in $[1,7]$, it was shown how the increased values of sensitivity at the wavelength $254 \mathrm{~nm}$ can be obtained for ultraviolet photodiodes.

Using the previous experience, the mathematical model of the construction of silicon photodiode based on the epitaxial structure enabling to regulate the absorption edge of silicon in the long-wave range of spectrum is suggested in this research. This construction will allow obtaining the silicon photodiodes that have sensitivity only in the ultraviolet spectral range.

The construction of a random photodiode is calculated using the system of inequalities that includes the following parameters:

$h$ - propagation depth of electric field due to the concentration gradient of charge carriers at the boundary "epitaxial silicon - low-resistance substrate";

$h_{e l}$ - thickness of epitaxial layer;

$x_{0}-$ length of the space charge region in epitaxial layer generated by the barrier between semiconductor and the conducting metal oxide layer.

$L_{p}$ - diffusion length of holes in epitaxial layer; 
$\alpha_{\lambda}-$ absorption coefficient of radiation with the given wavelength in semiconductor;

$d$-thickness of the silicon oxide layer;

$n$-refraction index of the metal oxide film;

$S_{I \varphi}-$ sensitivity caused by optical radiation falling on photodiode;

$I_{\mathrm{ph}}-$ specific value of photocurrent generated by a single plate providing sensitivity of photodiode at the given wavelength;

$P$ - power of incident optical radiation;

$S$ - area of photosensitive element.

It is obvious that, through regulating the thickness of the epitaxial layer, it is possible to regulate the longwave absorption edge of the photodiode structure on the whole. While developing such a structure, the main problem consists in accurate calculation of the absorption depth of the longest-wave radiation that is to be registered by the device.

It is shown in [1] that while providing certain constructive and technological requirements, it is possible to make photodiodes based on silicon with the value of current monochromatic sensitivity not lower than $0.1 \mathrm{~A} / \mathrm{W}$ at the wavelength $300 \mathrm{~nm}$ and the value $0.13 \mathrm{~A} / \mathrm{W}$ - at the wavelength $250 \mathrm{~nm}$ (Fig. 1). Photodiodes of the "Quantum" type are normalized at the wavelength $550 \mathrm{~nm}$. At this wavelength, the value of current monochromatic sensitivity amounts to $0.29 \mathrm{~A} / \mathrm{W}$, while at the maximum of spectral sensitivity it is $0.44 \mathrm{~A} / \mathrm{W}[1,9]$.

The disadvantage of silicon photodiodes, including those of the "Quantum" type, from the viewpoint of their application in ultraviolet radiometry, as it was mentioned above, consists in their sensitivity in the infrared spectral range.

Computer modeling shows that the suggested construction of a photodiode based at silicon epitaxial structure with thin basis can provide current monochromatic sensitivity at the wavelength $254 \mathrm{~nm}$ not lower than $0.05 \ldots 0.09 \mathrm{~A} / \mathrm{W}$. The results of modeling have been presented in Fig. 2. Here, only the part of sensitivity caused by the absorption depth of optical radiation with different wavelengths was considered [10].

Investigations carried out in [11] show that, in the operating range of wavelengths (200 to $500 \mathrm{~nm})$, practically total absorption of optical radiation occurs (up to $86 \%$ of radiation fallen on the semiconductor layer) in the layer with the thickness close up to $1.5 \mu \mathrm{m}$. The discrepancy between the calculation data (Fig. 2) can be explained by the fact that in the case of calculation, only absorption of optical radiation in silicon was taken into account without considering the properties of material, antireflection coating and many other.

Being based on the research, the conclusion can be made that the construction of photodiode including the epitaxial structure of $n^{+}$- $n$-type, in which the highresistance layer of $n$-type, located on the low-resistance substrate, is the operation layer (where absorption of optical radiation occurs).

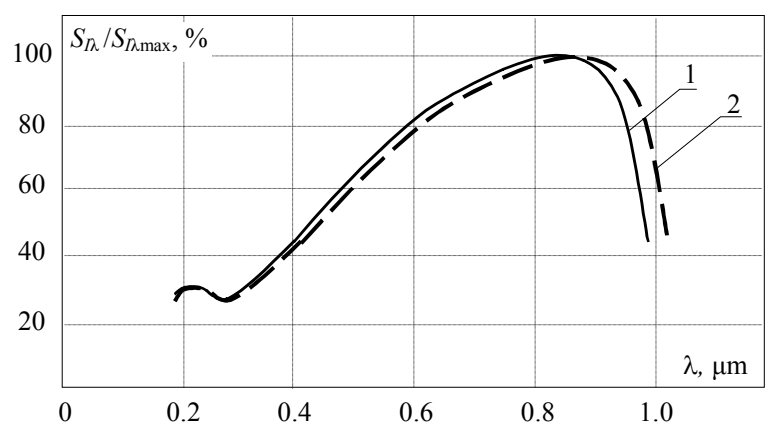

Fig. 1. Relative spectral characteristics of sensitivity of silicon photodiodes. 1 - "Quantum"; 2 - "Quantum-1".

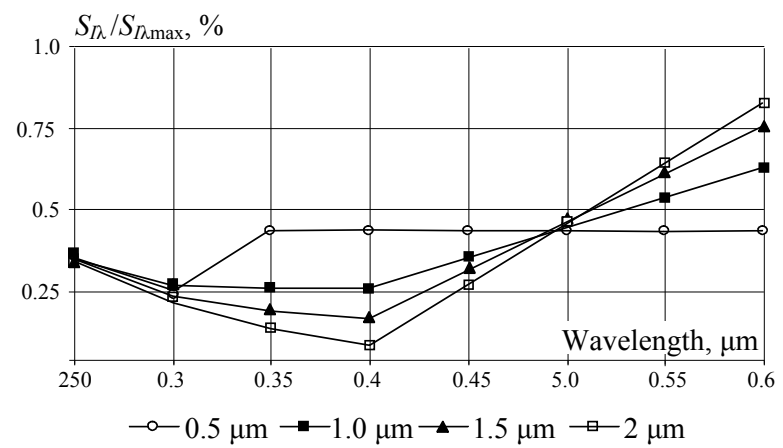

Fig. 2. Calculated values of relative spectral characteristics of sensitivity of silicon photodiodes based on epitaxial structures of different thicknesses.

To determine the highest possible value of sensitivity that can be obtained in this case, we shall use the approach described in $[6,7]$. Here, to provide maximal sensitivity of the photodiode structure, it is suggested to solve the system of inequalities that includes the main parameters of the photodiode construction and physical parameters of material.

The main contribution to the photo-signal in this structure is made by minority carriers, thus, the $L_{p}$ value will be determinative in the structure $n-n^{+}$. It should be mentioned that not only $L_{p}$ determines the depth of the region for collection of photo-generated charge carriers, but also the length of the space charge region in epitaxial layer $x_{0}$, generated by the barrier between $n$ and $p$ regions forming the $p$ - $n$ junction in the epitaxial layer.

The concentration of dopants in the epitaxial layer of $h_{e l}$ thickness exponentially decreases moving from the boundary "substrate - epitaxial layer" as a result of autodoping processes [11]. Thus, it contains electrical field caused by the distribution profile of dopant concentration [12]. The depth of propagation of this field should be also considered while calculating $h_{e l}$. Therefore, the thickness of the epitaxial layer can be determined using the following inequality:

$x_{0}<h_{e l} \leq\left(L_{p}+x_{0}+h\right)$. 
It is evident that the low-resistance substrate of the epitaxial structure should not affect sensitivity of the device. This requirement is provided by inequality (2):

$L_{p}<\left(h_{e l}-x_{0}\right)$.

To provide collection of charge carriers generated by short-wave optical radiation, it is necessary that the length of the space charge region $x_{0}$ in the highresistance layer should be longer than the absorption depth of this radiation. This absorption depth, in its turn, is determined by the absorption coefficient of this radiation $\alpha_{\lambda}$ (in $\mathrm{cm}^{-1}$ ) and is shorter than the diffusion length of minority carriers (in our case - holes). These requirements are provided by the inequality:

$\alpha_{\lambda}<x_{0} \leq L_{p}$.

Uniting the suggested expressions into a system enables to optimize the construction of epitaxial silicon photodiodes that operate in the photovoltaic mode to guarantee maximal sensitivity:

$\left\{\begin{array}{l}x_{0}<h_{e l} \leq\left(L_{p}+x_{0}+h\right), \\ L_{p}<\left(h_{e l}-x_{0}\right), \\ \alpha_{\lambda}<x_{0} \leq L_{p} .\end{array}\right.$

The first expression determines the region of collecting the carriers photo-generated by the long-wave component of the region of photodiode spectral sensitivity; the second one provides isolation of the highresistance epitaxial layer from the impact of charge carriers generated in the low-resistance substrate of the epitaxial structure; the third expression provides contribution of the short-wave component of the spectrum.

To guarantee maximal sensitivity of the photodiode, in addition to the above mentioned procedures, it is necessary to consider the reflection phenomenon of the optical radiation flux from the surface of the optically transparent antireflection coating of silicon dioxide.

The right choice of silicon dioxide thickness enables to minimize losses in photocurrent, related to the fact that a part of the optical radiation flux capable to generate minority charge carriers in semiconductors is reflected from both the surface of silicon dioxide and that of semiconductor. The thickness of the silicon dioxide layer $d$ is selected with account of the condition that the phase difference $\varphi$ of radiation at the round-trip passage through the layer is to be multiple $(2 n+1) \pi$. In the opposite case:

$d=\lambda(2 n+1) / 4$

In this case, the waves reflected from the boundaries "film - air" and "film - semiconductor" will be added in the antiphase and "suppress" each other [12].
The area of the photosensitive element should be also taken into account, as the value of photocurrent, provided that the whole plate of photodiode is illuminated by the radiation flux $P$, can depend on it.

$I_{\mathrm{ph}} \cong \frac{S_{I \varphi} P}{S}$ or $I_{\mathrm{ph}} S \cong S_{I \varphi} P$.

Thus, the system of inequalities (4) can be transformed into the system of inequalities and equations:

$$
\left\{\begin{array}{l}
x_{0}<h_{e l} \leq\left(L_{p}+x_{0}+h\right), \\
L_{p}<\left(h_{e l}-x_{0}\right), \\
\alpha_{\lambda}<x_{0} \leq L_{p}, \\
d=\lambda(2 n+1) / 4, \\
I_{\mathrm{ph}} S \cong S_{I \varphi} P .
\end{array}\right.
$$

The value of current monochromatic sensitivity at a certain wavelength can be also assessed using the results obtained in [13]:

$$
S_{I \lambda}=\left(1-R_{1 \lambda}\right) \frac{e_{0} \gamma_{\lambda} \lambda}{h c}\left(1-e^{-\alpha_{\lambda} x_{0}}\right) \text {, }
$$

where $S_{I \lambda}$ is the current monochromatic sensitivity for the given wavelength; $R_{1 \lambda}$ - coefficient of reflection from the surface "air - oxide - semiconductor" for the given wavelength; $\lambda-$ given wavelength; $\gamma_{\lambda}-$ photocurrent utilization coefficient (inner photoresponse); $h$ - Planck constant; $c$ - speed of light in vacuum; $e$ - elementary electric charge.

To reach that, (8) should be divided by the thickness of the metal oxide film:

$$
\begin{aligned}
& S_{I \lambda}=\left(1-R_{1 \lambda}\right) \frac{e_{0} \gamma_{\lambda} \lambda}{h c}\left(1-e^{-\alpha_{\lambda} x_{0}}\right) / d= \\
& =\left(1-R_{1 \lambda}\right) \frac{4 e_{0} \gamma_{\lambda}}{h c(2 n+1)}\left(1-e^{-\alpha_{\lambda} x_{0}}\right) .
\end{aligned}
$$

Analysis of the obtained equations and inequalities (7) and (9) performed by means of MS Office Excel 2003 showed (Fig. 3) that the maximal values of sensitivity at the wavelength $250 \mathrm{~nm}$ can reach 0.08 $0.09 \mathrm{~A} / \mathrm{W}$, if the epitaxial layer thickness is $1.5-2 \mu \mathrm{m}$. The calculation was based on the values $\alpha_{\lambda}, \gamma_{\lambda}, R_{1 \lambda}$ and $n$ presented in [13]. The value $x_{0}$ was assessed in $0.1-$ $0.3 \mu \mathrm{m}$, it accounting the fact that the photodiode should operate in the photovoltaic mode without electrical bias. The length of the space charge region in this case is conditioned by the potential barrier between $p$ and $n$ layers and epitaxial silicon substrate.

The analysis results prove that the best values of sensitivity in the ultraviolet spectrum can be obtained at the epitaxial layer $1.5-\mu \mathrm{m}$ thick. It is a complicated technological task requiring accurate equipment that enables to form $p-n$ junction with the accuracy close to $0.1 \mu \mathrm{m}$. 


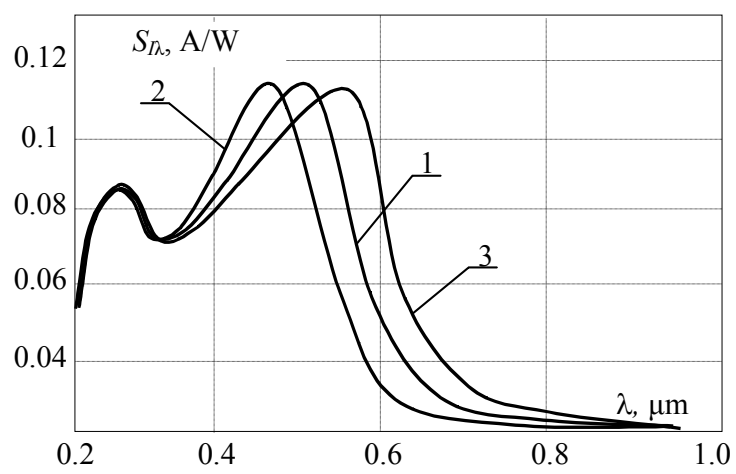

Fig. 3. Dependence of the calculated values of the current monochromatic sensitivity of silicon photodiode on the epitaxial layer thickness: $h_{e l}=1 \mu \mathrm{m}(1), 1.5 \mu \mathrm{m}(2), 2 \mu \mathrm{m}$ (3).

The best of the well-known analogues possess the current monochromatic sensitivity value $0.08 \ldots 0.07 \mathrm{~A} / \mathrm{W}$ at the wavelength $250 \mathrm{~nm}$. However, these are the devices based on gallium phosphide and zinc selenide. These materials are more expensive than epitaxial silicon. On the other hand, technological processes on silicon are generally more manufacturable, more well-tested in serial production, which provides a possibility for a low price for these photodiodes in batch production.

It should be indicated that the suggested report does not consider these characteristics influencing sensitivity photodiode, as the surface-state density on the surface of silicon epitaxial structure. The analytical consideration of this factor demands further investigation.

\section{Conclusions}

The mathematical model for calculating the value of current monochromatic sensitivity of silicon photodiode based on epitaxial structure for operation in the ultraviolet spectral range has been developed. The suggested model enables to calculate the construction having low sensitivity for the wavelengths longer than $600 \mathrm{~nm}$ and maximal values at the wavelength $254 \mathrm{~nm}$.

\section{References}

1. Yu.G. Dobrovolskii, V.V. Riukhtin, A.B. Shimanovskii, Silicon p-n photodiodes for near ultraviolet spectral range // Tekhnologia konstruirovanie $v$ elektronnoi apparature, No.4-5, p. 44-46 (2001), in Russian.
2. A.A. Ashcheulov, Yu.G. Dobrovolskii, B.M. Godovaniuk, Optimization of parameters of silicon epitaxial structures to provide radiation resistance of photodiodes based on them // Naukovyi visnyk Chernivetsk. Universitetu, 30, p. 170-174 (1998), in Ukrainian.

3. A.A. Ashcheulov, Yu.G. Dobrovolskii, B.M. Godovaniuk, Radiation resistance of photodiodes based on silicon epitaxial structures // Naukovyi visnyk Chernivetsk. Universitetu, 32, p. 107-109 (1998), in Ukrainian.

4. A.I. Malik, G.G. Grushka, Optoelectronic properties of heterojunctions metal - gallium phosphide oxide // Fizika tekhnika poluprovodnikov, 25(10), p. 18911695 (1991), in Russian.

5. A. Malik, A. Seco, E. Fortunator, R. Martins, B. Shabashkevich, S. Piroszenko, A new high ultraviolet sensivity FTO-GaP Schottky photodiode fabricated by spray pyrolysis // Semicond. Sci. Technol. 13, p. 102-107 (1998).

6. Yu.G. Dobrovolskii, Photodiode on the basis of gallium phosphate with the promoted sensitivity in short-wave range of UV spectrum // Tekhnologia konstruirovanie $v$ elektronnoi apparature, No.5, p. 31-34 (2012), in Russian.

7. Yu.G. Dobrovolskii, L.Y. Podkamin, G.V. Prohorov, Photodiode based on gallium phosphate with the promoted sensitiveness at the wavelength $250 \mathrm{~nm} / /$ 10th Intern. Conf. on Correlation Optics (Correlation Optics'11), Черновцы, 2011; http://www.itf.cv.ua/ corropt11/Data.files/programme.htm

8. Photodiodes of the "Quantum" type, Technical characteristics); http://ckb-rhythm.narod.ru/ fdSipn_fdkvant1.htm

9. V.F. Gremeniuk, M.S. Tivanov, V.B. Zalesskii, Solar Cells Based on Semiconductor Materials. BGU Publ. Centre, Minsk, 2007.

10. Yu.G. Dobrovolskii, E.B. Komarov, M.P. Biksei, Two-spectrum photodetector // Tekhnologia konstruirovanie $v$ elektronnoi apparature, No.3, p. 18-22 (2005), in Russian.

11. V.L. Bonch-Bruyevich, L.G. Kalashnikov, Physics of Semiconductors. Nauka, Moscow, 1977 (in Russian).

12. D.V. Sivukhin, General Course of Physics. In 5 volumes. V. IV. Optics. 3-rd ed. Fizmatlit Publ., 2005, p. 426-430 (in Russian).

13. M.P. Biksei, Yu.G. Dobrovolskii, B.G. Shabashkevich, Photodetector of ultraviolet radiation on the basis of gallium phosphide // Prikladnaia fizika, 4, p. 97-100 (2005), in Russian. 